\title{
Intégration de l'hygiène dans les projets d'eau et d'assainissement exécutés par les ONG nationales au Mali
}

\section{Integration of hygiene in water and sanitation projects carried out by national NGOs in Mali}

\author{
Touré $\mathrm{O}^{1^{*}}$, Dembélé $\mathrm{B}^{2}$, Maiga $\mathrm{OS}^{3}$, Kanté $\mathrm{N}^{4}$, Diakité $\mathrm{C}^{5}$, Niangaly $\mathrm{A}^{6}$
}

1,4,5,6Institut National de Recherche en Santé Publique, ${ }^{2}$ Consultant ONG ADDA, ${ }^{3}$ Agence Nationale de la Sécurité Sanitaire des Aliments

\section{Auteur correspondant : oussou_toure@hotmail.com}

\section{RESUME}

Introduction : La présente étude avait pour but d'apprécier la prise e compte de l'hygiène dans les projets d'eau et d'assainissement exécutés par les ONG nationales au Mali. Méthodologie : La Politique et des Normes et Procédures en matière d'hygiène publique ont servi de référence pour apprécier les services fournis par les ONG aux bénéficiaires. La qualité des services a été appréciée par observation des infrastructures. Résultats : Moins de $3 \%$ des concessions avaient leurs latrines à moins de 15 mètres du puits d'eau familial ; 65 $\%$ des points d'eau publics étaient équipés d'infrastructures d'assainissement (puisard, lavoir, etc..). Seulement $10 \%$ des concessions avaient de dispositifs de lavage des mains à la sortie des latrines (présence d'un récipient contenant de l'eau et du savon). Aussi $94 \%$ des latrines scolaires étaient équipées de Kit de lavage des mains, mais seulement $23,5 \%$ contenaient de l'eau. La moitié des latrines vues était propre, $70 \%$ dégageaient de mauvaises odeurs, et $60 \%$ étaient sans couvercle. Tous les points d'eau publics étaient clôturés. Conclusion : Les conditions de promotion de l'hygiène n'étaient pas toujours réunies autour des ouvrages réalisés par les ONG locales

Mots clés: Hygiène, projets AEPA, ONG local, Mali

\section{ABSTRACT}

Introduction: The main aim of this research is to appreciate how far hygiene has been taken into account in water supply and sanitation projects implemented by local NGO in rural Mali. Method: Hygiene national policies, standards and procedures had been considered has a reference to appreciate services provided to beneficiaries by local NGO. Services quality has been appreciated on the ground by infrastructures observations. Results: Less than $3 \%$ of households had their latrines at a distance less than $15 \mathrm{~m}$ from the family water supply well; $65 \%$ of public water supply sources were equipped with sanitation infrastructures (sink, washing plant, ..). Only $10 \%$ of households had handwashing kit next to latrines (presence of utensil containing water and soap). Also, 94 $\%$ of school latrines were equipped with a hand washing kit; but only $23.5 \%$ of them contained water. A half of observed latrines was clean; $70 \%$ them smell but and 60 $\%$ had not a cover. All public water sources had fence. Conclusion: Hygiene promotion conditions were not always met in water supply projects implemented by local NGO's in rural Mali.

Key words: Hygiene, WAS project, local NGO, Mali.

\section{INTRODUCTION}

L'accès à une eau de boisson saine et à des infrastructures d'assainissement appropriées, est reconnu comme un droit humain par les Nations Unies. Par conséquent, la satisfaction de ces besoins de base est une obligation des gouvernants vis-à-vis de leurs citoyens. C'est en réponse à cet impératif que les Gouvernements successifs de ces dernières années ont retenu l'amélioration de l'AEPHA ? parmi leurs priorités de développement, et ont souscris aux ODM ? 2000-2015. Les efforts considérables déployés par les pouvoirs publics et locaux, les partenaires au développement et société civile, pour combler le déficit important en besoins en AEPHA conformément aux engagements nationaux relatifs à ces ODM, ont abouti à des résultats très significatifs en termes de satisfaction des besoins en eau potable notamment (1) .

Pour maximiser ces résultats, il est indispensable de promouvoir l'hygiène individuelle et collective, particulièrement autour des infrastructures elle mêmes. Aussi, l'intégration d'un volet hygiène dans le projet d'approvisionnement en eau potable ou d'assainissement figure en bonne place dans la politique nationale du secteur eau-assainissement $(1,2,3)$.

Dans sa volonté de concrétiser cette disposition, l'Etat a bénéficié d'un soutien constant des partenaires techniques et financiers, au nombre desquels Wateraid Mali (WAM), une Organisation Non Gouvernementale (ONG) Internationale, engagée aux côtés des acteurs nationaux depuis plus d'une décennie. Elle intervient dans tous les axes stratégiques, avec un effort singulier sur la fourniture des services de base en s'appuyant sur les ONG locales (ONGL) spécialisées dans le secteur (14). Pour optimiser les interventions de ces dernières, WAM a soutenu la mise en place du GP/EHA dont une des missions principales, est le renforcement des capacités de ses membres pour une plus forte contribution à l'accès des communautés encadrées aux services d'AEPHA.

Mais, très peu d'investigations ont été conduites pour apprécier le niveau pris en compte de l'hygiène dans les projets d'approvisionnement et d'assainissement exécutés sur le terrain, notamment les acteurs les plus proches des communautés à savoir les ONGL ;

La présente étude entre dans ce cadre. La mission, initiée par le GP/EHA et soutenue financièrement par WAM envisage de mener une étude visant l'appréciation du niveau de prise en compte de l'hygiène par les différents 
acteurs de terrain, notamment les ONGL membres, pour assurer des services aux plus pauvres.

\section{METHODOLOGIE}

Site et période de l'étude

L'étude a eu lieu du 15 Octobre et 30 Novembre 2015 dans les régions de Koulikoro, Ségou et Mopti, zones couvertes par les ONGL membres du PG/EHA.

\section{Echantillonnage}

Sélection des localités : c'est un choix raisonné tenant compte de la représentativité en terme de répartition des infrastructures par catégorie; ainsi, chaque fois qu'une ONGL intervient en milieu rural ou urbain, le centre urbain est retenu avec des sites ruraux proportionnellement à l'importance des réalisations dans les différents sites ; Lorsqu'une I'ONGL n'intervient qu'en milieu rural ou en milieu urbain uniquement, les sites sont retenus en conséquence en tenant compte du type d'infrastructures ;

- Choix des infrastructures :

- Ouvrages collectifs (ouvrages hydrauliques et latrines publiques) : Vu leur faible concentration, ce choix aussi a été raisonné, en tenant compte de la répartition spatiale des ouvrages et du type ; par exemple, dans le cas des latrines publiques il a été tenu compte de la représentativité des latrines scolaires et des latrines situées dans d'autres lieux (marchés, lieux de culte, etc...), et pour ce qui concerne les ouvrages hydrauliques il a été tenu compte de leur typologie.

- Ouvrages individuels, les latrines familiales : ici, le milieu rural a été ciblé. Le choix des ménages à enquêter a été fait sur une base quasi aléatoire ; sur la base d'une liste des ménages dressée par l'animateur, cinq ménages ont été tirés au hasard.

\section{Collecte des données}

Suite à la revue des documents de Politique, stratégie ou de planification des différents acteurs, il été dressé une liste de critères tirés des normes d'hygiènes retenues par le Ministère de la santé. Chaque critère été apprécié par des indicateurs appropriés. Ainsi, une fiche collecte des données a été élaborée pour chaque type d'ouvrage.

\section{Traitement des données}

Les données de caractérisation des ouvrages ont été saisies et traitées et analysées sur le logiciel SSP pour estimer les indicateurs d'appréciation de chaque critère retenu et tirer les conclusions. Pour ce critère, les indicateurs varient selon le type d'infrastructures.

\section{RESULTATS}

Protection de l'environnement

Il s'agit de mesures prises pour éviter la contamination de l'environnement par une pollution provoquée par l'exploitation des ouvrages.

\section{- Latrines familiales}

Pour ce type d'ouvrage l'aspect le plus important, c'est la distance entre la latrine et un point d'eau situé dans les environs.

Dans $57 \%$ des ménages enquêtés les latrines sont situées à plus de 15 mètres du point d'eau familial. Dans $40 \%$ des ménages enquêtés, il n'y a pas de point d'eau à l'intérieur de la cour ; donc il n'y a aucun risque potentiel. Seuls 3\% des latrines familiales étaient situées à moins de $15 \mathrm{~m}$ d'une source d'eau.

\section{- Points d'eau publics}

Dans ce cas, suivant le type d'ouvrage ou de son usage, plusieurs équipements concourent à l'assainissement de l'environnement. Ainsi, les points d'eau, selon leurs usages ont été équipés d'infrastructures d'assainissement adaptées.

Les points d'eau visités étaient complétés par des infrastructures secondaires adaptés aux besoins d'usage et d'assainissement, dont $65 \%$ pour les puisards, $14 \%$ pour les puisards et lavoirs, et $7 \%$ pour le puisards et lavoirs et abreuvoirs. Ces équipements se rencontraient surtout près des PM. Seulement $14 \%$ en étaient dépourvus.

\section{Lavage des mains à la sortie de latrines \\ - Latrines familiales}

Dans ce cas l'appréciation porte sur les dispositions relatives à l'hygiène individuelle et la propreté de l'environnement.

Un récipient contenant de l'eau pouvant servir au lavage des mains à la sortie des latrines était absent dans les ménages visités. Cependant des morceaux de savons ou de récipient contenant de la cendre ont été retrouvés à l'intérieur des enceintes des latrines respectivement dans $(10 \%)$ et $(13,3 \%)$ (Tableau 1), (Images 1 et 2 ).

\section{- Latrines publiques}

La plupart des latrines publiques, scolaires sont équipées de kits de lave mains (17/18 soit 94\%), mais peu de réservoirs contenaient de l'eau indispensable à la pratique du lavage des mains (Image 3) ; l'eau est disponible dans seulement 4 réservoirs sur 17 observés (23,5\%), (Image 4). Dans un cas il n'y avait pas de récipient.

\section{Propreté}

Ce critère a été évalué par la présence de souillure, de mouches et d'odeurs sur la dalle de la latrine. Le devenir des eaux usées (en général eau de bain et urine) est aussi un aspect important.

\section{- Latrines familiales}

Sur 30 ménages enquêtés, seules 4 latrines familiales $(13,3)$ n'étaient pas fonctionnelles. II s'agit soit de latrines totalement remplies, donc abandonnées, soit de latrines effondrées, donc inutilisables. Des souillures étaient visibles sur les dalles de 13 latrines fonctionnelles, soit $50 \%$, et aucune souillure n'a été observée dans 13 autres $(50 \%)$.

Les $70 \%$ des latrines observées dégageaient de mauvaises odeurs avec une présence de mouches. Par 
ailleurs, les trous de défécation de $60 \%$ ne disposent pas de couvercle ; ce qui facilite l'accès des mouches et autres vecteurs aux excréta.

Dans la majorité des cas (24 sur 30 , soit $80 \%$ ), les eaux usées sont drainées vers des puisards, mais hors de la cour ; une seule latrine était raccordée à un puisard à l'intérieur de la cour. Dans 17\% (5/25) des cas il n'y avait pas d'écoulement.

\section{- Points d'eau}

$67,9 \%$ des points d'eau enquêtés étaient clôturés, notamment les PMH et les PM. 32,1\% des points d'eau ne comportaient pas de clôture ; ces derniers étaient constitués généralement de bornes fontaines et de certains puits traditionnels (Tableau 2), (Image 5).

\section{DISCUSSION \\ Protection de l'environnement \\ - Latrines}

Dans $97 \%$ des concessions visitées, les latrines étaient situées à plus de $15 \mathrm{~m}$ du point d'eau familial, lorsqu'il y en a. Le risque de contamination était probable seulement dans $3 \%$ des cas. L'emplacement des latrines familiales respectait la distance réglementaire (7).

\section{- Points d'eau}

$67,9 \%$ des points d'eau visités étaient clôturés ; $86 \%$ étaient complétées par des infrastructures dédiées à d'autres usages de l'eau que la boisson (lavoir ou abreuvoir) reliées à des ouvrages d'assainissement (puisard). $(2,11)$

\section{Hygiène}

\section{- Latrines}

Un récipient contenant de l'eau pouvant être utilisé pour laver les mains à la sortie d'une latrine n'était disponible dans aucun des ménages visités ; Cependant des morceaux de savons ont été trouvés dans $10 \%$ des latrines et des récipients contenant de la cendre dans $13,3 \%$ des latrines. Ces dispositifs étaient utilisés pour les bains individuels. Un réservoir a été observé devant $94 \%$ des latrines scolaires visitées, mais $23,3 \%$ seulement de ces réservoirs contenaient de l'eau, garantissant effectivement la pratique du lavage des mains à la sortie des toilettes. (11)

Des souillures ont été observées sur les dalles ou trou de défécation de $50 \%$ des latrines familiales observées ; 70 $\%$ dégagaient des mauvaises odeurs et des mouches pullulaient tout autour ; enfin $60 \%$ des trous de défécation avaient des couvercles défectueux ou étaient non couverts. Les risques de contamination étaient très élevés (12).

\section{- Points d'eau}

Les aires d'assainissement de l'ensemble des points d'eau modernes (PMH, PDG et BF) étaient clôturées ou délimités facilitant ainsi leur entretien. Ces ouvrages prennaient en compte les mesures de protection de l'environnement $(8,11,12)$.

\section{CONCLUSION}

Le lavage des mains au savon ne semble pas être pratiqué dans les ménages. Et les conditions de la pratique ne sont pas réunies en beaucoup d'endroits des lieux publics.

\section{Références}

1- ALTAF M.A., et HUGUES J.A., (1994), « Mesurer la demande pour des services améliorés d'assainissement urbain: Résultats d'une étude d'évaluation contingente à Ouagadougou, Burkina Faso», Urban Studies, Vol. 31, No. 10, 1994, p.1763-1776.

2- BRIAND A., LARE L. A., (2011), Consentement à payer pour l'amélioration de l'accès à l'eau potable des ménages Bamakois et Ouagalais, CREAM, Université de Rouen,

3- BRIAND A., LARE L. A., (2013), « Les déterminants de la demande domestique de raccordement auprès des Petits Opérateurs Privés en eau potable : Cas des quartiers périphériques de Maputo», Revue économique 64, p.685-719.

4-BIAND, L., « L'accès à l'assainissement amélioré dans les quartiers précaires de Ouagadougou », L'Actualité économique, Revue d'analyse économique, vol. 94, no 3 , septembre 2018, 362

5- CLOHOUNTO, J., DEDJINOU, S. (2012) « les bénéfices d'adduction d'eau potable dans la vallée de l'Oueme », Unversité de Cotonou, Benin $19 p$.

6- ESREY S. A., FEACHEM R., HUGUES J., (1985), « Interventions pour la lutte contre les maladies diarrhéiques chez les jeunes enfants: amélioration de l'approvisionnement en eau et des installations d'élimination des excreta», Bulletin of the World Health Organization 63 (4): p.757-772.

7- ESREY S., (1996), « Eau, déchets et bien-être: une étude multi pays. American Journal of Epidemiology», 143: p.608-623.

8- ESREY S., HABICHT J.-P., (1986), « Les preuves épidémiologiques pour la santé bénéficient d'une amélioration de l'eau et de l'assainissement dans les pays en développement», Epidemiologic Reviews 8: p.117-128. 9- ESREY S., POTASH, J., ROBERTS L., SHIFF C., (1991), « Effets de l'amélioration de l'approvisionnement en eau et de l'assainissement sur l'ascaridiase, la diarrhée, la dracunculose, l'ankylostomiase, la schistosomiase et le trachome», Bulletin of the World Health Organization 69: p.609-621.

10- ESREY S., HABICHT J.-P., CASELLA G., (1992), « L'effet complémentaire des latrines et l'augmentation de l'utilisation de l'eau sur la croissance des nourrissons dans les zones rurales du Lesotho», American Journal of Epidemiology, 135: p.659-666.

11- FEWTRELL L., KAUFMANN R., KAY D., ENANORIA W., HALLER L., et COLFORD JR.J, (2005), " Interventions dans le domaine de l'eau, de l'assainissement et de l'hygiène pour réduire la diarrhée dans les pays moins développés: revue systématique et méta-analyse», The Lancet Infectious Desease 5 (1), p.42-52. 
12- JENKINS, M.-W., Curtis, V. (2005) Achieving the "good life": why some people want latrines in rural Benin. Social Science and Medicine 61: 2446-2459.

13- LARE, L.-A. (2010), « Analyse de la demande en assainissement amélioré des ménages : le cas des latrines écologiques à Dapaong au Nord du Togo ", CREAM, Université de Rouen.

14- SERAJ B. F. K., (2008), "Willingness to Pay for Improved Sanitation Services and its Implication on Demand Responsive Approach of BRAC Water, Sanitation and Hygiene Programme», Working Paper No. 1 BRAC JMP WHO/UNICEF JMP, «Progress on Drinkingwater and Sanitation», 2012 Update

15- TRAORE, K. (2017). De la Sensibilisation des Populations à la Gestion de l'Environnement Urbain dans les Quartiers Précaires De la Ville d'Abidjan. Etude de la Population Africaine Vol. $22 N^{\circ} 2$, p.154-173.

16- WHITTINGTON D., BRISCOE J., MU X., BARRON W., (1990a), « Estimation de la volonté de payer pour les services d'eau dans les pays en développement: une étude de cas sur l'utilisation des enquêtes d'évaluation contingentes dans le sud de Haiti», Economic Development and Cultural change, 38, (2), p.293-311. 17- WHITTINGTON D., LAURIA D.T., CHOE K., HUGHES J.A., SWARNA V., WRIGHT A.M., (1993), " L'assainissement domestique à Kumasi, Ghana: une description des pratiques, attitudes et perceptions actuelles», World Development 21, p.733-748. ??? $4 / 05 / 2018$

18- TIOGANG, D.- ; M.-M. ; Thérèse, F.- M. ; Assoumou, E.- E. \& Wabo, A. (2008). Valeur économique de l'amélioration de l'approvisionnement en eau potable des zones rurales au Cameroun. TROPICULTURA, 26, 4, p.224-228

\section{Liste des tableaux et images}

Tableau 1 : Disponibilité de savon/désinfectant dans les latrines familiales

\begin{tabular}{lcc}
\hline Disponibilité de désinfectant & Effectifs & Pourcentage \\
\hline Savon & $\mathbf{3}$ & $\mathbf{1 0 , 0}$ \\
Cendre & 4 & $\mathbf{1 3 , 3}$ \\
Aucun & $\mathbf{2 3}$ & $\mathbf{7 6 , 7}$ \\
Total & $\mathbf{3 0}$ & $\mathbf{1 0 0 , 0}$ \\
\hline
\end{tabular}

Source : enquête de terrain, étude techniques, technologies et stratégies WASH

Tableau 2: Présence de clôture

\begin{tabular}{lcc}
\hline Présence de clôture & Effectifs & Pourcentage \\
\hline Oui & 19 & 67,9 \\
Non & 9 & 32,1 \\
Total & $\mathbf{2 8}$ & $\mathbf{1 0 0 , 0}$ \\
\hline
\end{tabular}

Source : enquête de terrain, étude techniques, technologies et stratégies WASH 


\section{MALI SANTE PUBLIQUE 2020 SOMASAP}

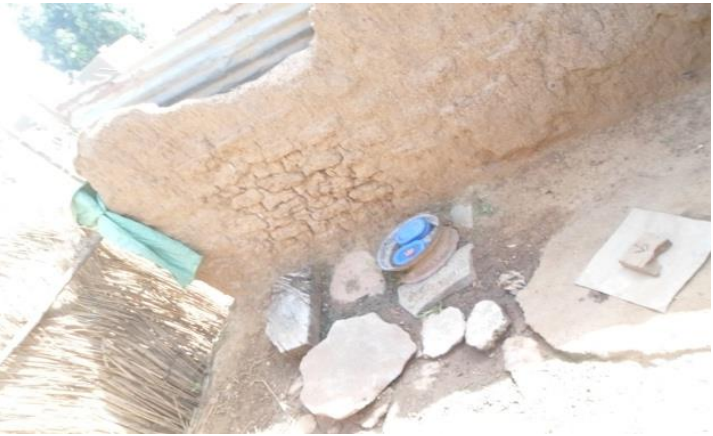

Image 1: intérieur d'une latrine avec du savon

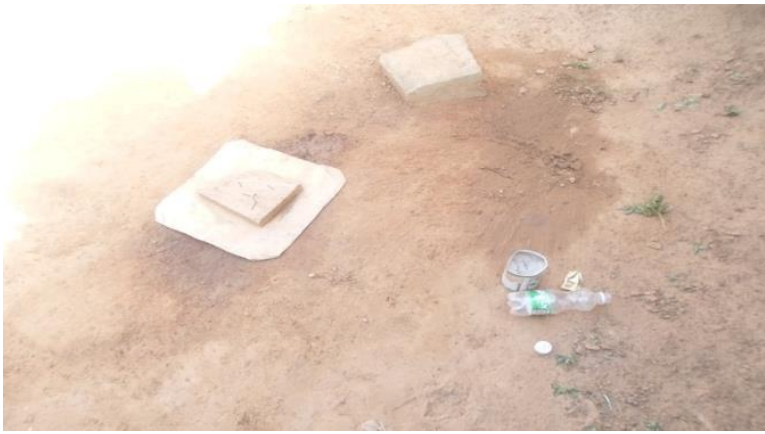

Image 2 : intérieur d'une latrine avec du cendre

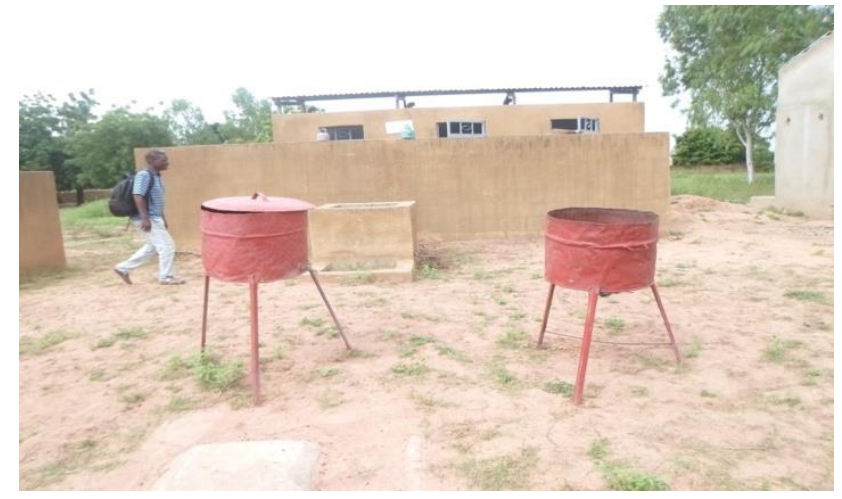

Image 3: Réservoir sans eau devant des latrines

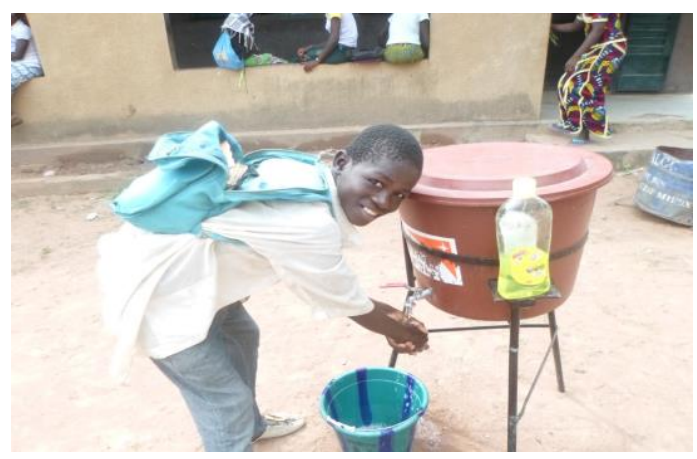

Image 4 : Dispositif de lavage de main complet en

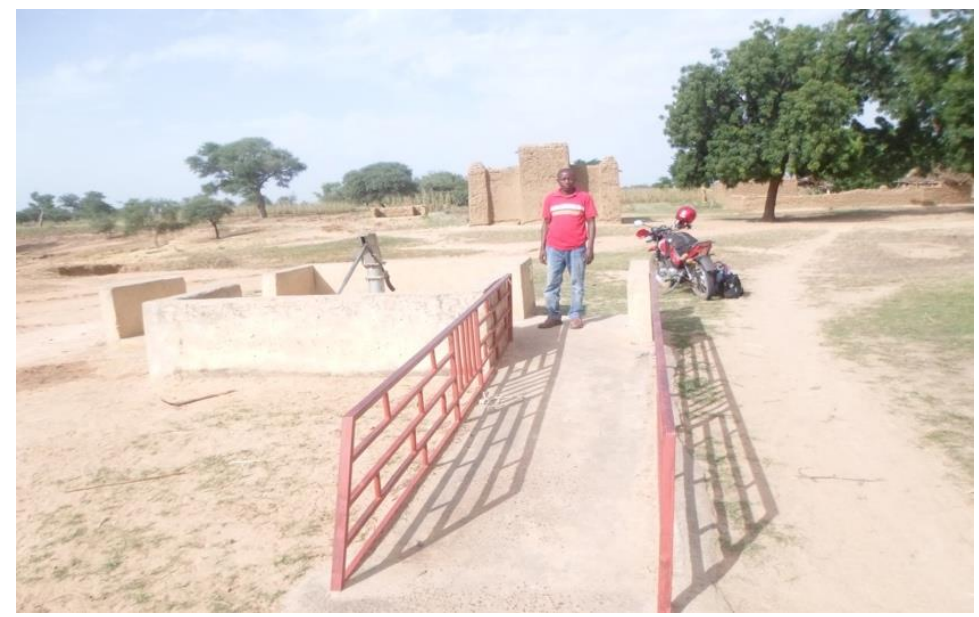

Image 5 : Clôture de PHM avec rampe d'accès pour handicapés 